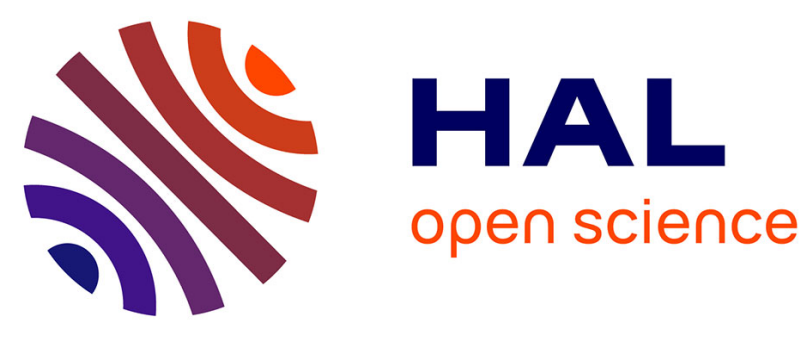

\title{
Terahertz amplifier based on gain switching in a quantum cascade laser
}

\author{
Nathan Jukam, Sukhdeep Dhillon, Dimitri Oustinov, Julien Madéo, \\ Christophe Manquest, Stefano Barbieri, Carlo Sirtori, Suraj P. Khanna, \\ Edmund H. Linfield, Alexander Giles Davies, et al.
}

\section{To cite this version:}

Nathan Jukam, Sukhdeep Dhillon, Dimitri Oustinov, Julien Madéo, Christophe Manquest, et al.. Terahertz amplifier based on gain switching in a quantum cascade laser. International Quantum Cascade Lasers School \& Workshop, 2010, Florence, Italy. pp.715-719, 10.1038/nphoton.2009.213. hal-00519407

\section{HAL Id: hal-00519407 https://hal.science/hal-00519407}

Submitted on 20 Sep 2010

HAL is a multi-disciplinary open access archive for the deposit and dissemination of scientific research documents, whether they are published or not. The documents may come from teaching and research institutions in France or abroad, or from public or private research centers.
L'archive ouverte pluridisciplinaire $\mathbf{H A L}$, est destinée au dépôt et à la diffusion de documents scientifiques de niveau recherche, publiés ou non, émanant des établissements d'enseignement et de recherche français ou étrangers, des laboratoires publics ou privés. 


\title{
Terahertz amplifier based on gain switching in a quantum cascade laser
}

\author{
Nathan Jukam ${ }^{1}$, Sukhdeep S. Dhillon ${ }^{1}$, Dimitri Oustinov ${ }^{1}$, Julien Madeo ${ }^{1}$, Christrophe \\ Manquest $^{2}$, Stefano Barbieri ${ }^{2}$, Carlo Sirtori ${ }^{2}$, Suraj P. Khanna ${ }^{3}$, Edmund. H. Linfield ${ }^{3}$, A. \\ Giles Davies ${ }^{3}$, and Jérôme Tignon ${ }^{1}$ \\ ${ }^{1}$ Laboratoire Pierre Aigrain, Ecole Normale Supérieure, UMR 8551 CNRS, UPMC Univ. Paris 6 \\ 75005 Paris, France \\ ${ }^{2}$ Matériaux et Phénomènes Quantiques, Université Denis Diderot - Paris 7, UMR 7162 CNRS, \\ 75013 Paris, France \\ ${ }^{3}$ School of Electronic and Electrical Engineering, University of Leeds, Leeds LS2 9JT, UK
}

Terahertz (THz) time domain spectroscopy (TDS) is widely used in a broad range of applications where knowledge of both the amplitude and phase of a $\mathrm{THz}$ wave can reveal useful information about a sample [1]. However, a means of amplifying THz pulses which would be of great benefit for improving the applicability of TDS is lacking. While THz quantum cascade lasers (QCL) [2] are promising devices for $\mathrm{THz}$ amplification [3], gain clamping [4] limits the attainable amplification [5]. Here we circumvent gain clamping and demonstrate amplification of THz pulses by ultrafast gain switching of a QCL via the use of an integrated Auston switch [6]. This unclamps the gain by placing the laser in a nonequilibrium state that allows large amplification of the electromagnetic field within the cavity. This technique offers the potential to produce high field THz pulses that approach the QCL saturation field. 
Terahertz (THz) time domain spectroscopy (TDS) is a powerful technique used to generate and detect pulses of broadband $\mathrm{THz}$ radiation [1]. It is now widely employed for THz imaging [7], non-destructive testing [8], and complex compound detection [9]. In order to generate $\mathrm{THz}$ pulses, near-infrared femtosecond lasers are used to excite photoconductive antennas [10] or nonlinear crystals [11]. Although the peak $\mathrm{THz}$ electric fields generated by these sources can be relatively high, the field amplitude per unit frequency is rather small owing to the extremely large spectral bandwidth of the generated $\mathrm{THz}$ pulse. Larger $\mathrm{THz}$ field amplitudes can be realised by increasing the power of the femtosecond laser. However, this requires large, complex and costly regenerative Ti:Sapphire amplifier systems [11]. A compact, practical and direct amplifier of $\mathrm{THz}$ pulses would therefore be of great interest.

A promising candidate for a $\mathrm{THz}$ amplifier is the recently realised $\mathrm{THz}$ quantum cascade laser (QCL) [2]. In this semiconductor-based source laser action takes place through electronic intersubband transitions [12]. Recently THz TDS has been used to measure the gain spectra of QCLs [3]. In these experiments, the QCL essentially acts as an amplifier of THz probe pulses that are transmitted through the laser. [13]. However, the amplification is limited by gain clamping, which fixes the gain to the sum of the waveguide and mirror losses during laser action. The amplification can be increased by reducing the mirror reflectivity through the use of anti-reflection (AR) coatings [3]. However, even for relatively high $\mathrm{THz}$ frequencies, low loss dielectric AR coatings are extremely challenging to realize [14] owing to the large coating thicknesses required. Tapered QCLs can also be used to amplify terahertz radiation [15]. However, the flared facets of tapered amplifiers are extremely wide and their output suffers from severe astigmatism [16]. This precludes their use for TDS, where high beam quality and a tight focus on the detector are essential to maximize the measured field. 
Here we employ the technique of gain switching [17, 18, 19], which consists in turning on the gain of a laser on a time scale that is much faster than the build up time of the laser field. During this period, the gain is greater than the total losses and unclamped from its value at threshold. The bare cavity gain of the laser (i.e. the un-clamped gain in the absence of a laser field) can therefore be accessed, and used to amplify injected $\mathrm{THz}$ probe pulses. In this paper, we demonstrate large $\mathrm{THz}$ pulse amplification (up to $26 \mathrm{~dB}$ excluding coupling losses) that is well above the limits imposed by the mirror losses and gain clamping. The gain switching is performed by an Auston switch [6] integrated into the QCL, where femtosecond illumination allows photo-excited carriers to change the switch's resistance from an insulating to a conductive state on an ultra-fast time scale.

The active region of the QCL used in our work is based on a bound-to-continuum design [20] which has been modified to lase at $2.4 \mathrm{THz}$. A schematic of the device is shown in figure 1a. Electrons from the top of the QCL ridge flow down through the active region to a highly doped $n++$ layer. From the $n++$ layer, electrons flow horizontally to two annealed AuGeNi contact layers on each side of the ridge. The integrated Auston switch was realized by etching an $80 \mu \mathrm{m}$ gap through one of the AuGeNi contact layers down to the semiinsulating GaAs substrate. Processed samples were cleaved into laser bars of $1.5 \mathrm{~mm}$ length.

An electrical schematic of the sample is given in figure 1b. The QCL can be represented as a capacitor in parallel with a shunt resistor. The lower $\mathrm{n}^{++}$layer can be treated as a resistance in series with the QCL, and the Auston switch as a capacitor that shorts when illuminated by a femtosecond laser. The QCL and Auston switch are independently biased with two external circuits which share a common ground. This permits the QCL to be biased just below threshold, which significantly reduces the current needed from the Auston switch to drive the laser above threshold. The Auston switch, QCL ridge, and the common ground are connected to external circuits with bond wires. When the femtosecond laser illuminates 
the Auston switch (ON state) the voltage of the common ground changes on an ultra-fast (picosecond) time scale. This drives an electrical pulse through an $\mathrm{RC}$ circuit formed by the resistive $\mathrm{n}^{++}$layer and the QCL's capacitance. At longer time scales the external circuit will restore the voltages on the device to their original values. Without femtosecond laser illumination the Auston switch is highly resistive and hence in the OFF state.

Figure 1c shows the characteristic light-current density (L-J) and voltage-current density (V-J) curves of the device at $7 \mathrm{~K}$ without illumination of the Auston switch. The laser threshold is $172 \mathrm{~A} / \mathrm{cm}^{2}$, and maximum power occurs at $184 \mathrm{~A} / \mathrm{cm}^{2}$. The negative differential resistance in the V-J curve corresponds to a drop in output power, owing to mis-alignment of the bandstructure [20].

Figure $1 \mathrm{~d}$ shows a schematic of the experimental arrangement. Broadband $\mathrm{THz}$ probe pulses are generated by discharging a photoconductive antenna with the same femtosecond laser that is used to excite the Auston switch. The THz probe pulses are then coupled into the QCL waveguide through the end facets. The transmitted pulses are measured using free-space electro-optic sampling (which is insensitive to the QCL laser emission [4]) in conjunction with a delay line for the femtosecond detection beam. Using a reference pulse, the amplification of the THz probe pulse and the gain of the THz QCL can be found [13]. Another delay line, for the femtosecond laser beam which activates the Auston switch, permits the time delay between Auston switch and the THz probe pulse to be varied. (Further details can be found in the experimental methods section).

Owing to the facet reflectivity, when the injected $\mathrm{THz}$ probe pulse reaches the output facet, part of the pulse is transmitted (single pass) and the remaining part is reflected back into the QCL cavity. The reflected pulse experiences a series of multiple passes through the cavity, and hence undergoes a longer amplification length. Figure 2 (a-d) shows the electric fields of the $\mathrm{THz}$ probe pulses transmitted through the QCL for 1, 3, 5 and 7 passes with the Auston 
switch ON (blue lines) and OFF (black lines). The corresponding spectra of the fields are shown in figures $2(\mathrm{e}-\mathrm{h})$. For each scan the time delay between the Auston switch and the $\mathrm{THz}$ probe pulse was adjusted to maximize the output pulse. The QCL is biased just below laser threshold, at a current density of $168 \mathrm{~A} / \mathrm{cm}^{2}$, in order to minimize the current that needs to be injected by the Auston switch.

For the single pass, with the Auston switch ON, we observe an increase in the number of field oscillations (figure 2a) and a larger spectral amplitude (figure 2e) compared to the Auston Switch OFF. Figures $2 \mathrm{~b}$ and $\mathrm{c}$ show the electric fields of output pulses that undergo multiple passes in the QCL (3 and 5 passes respectively). With the Auston switch OFF, the amplitude of the transmitted pulse decreases as the number of passes through the QCL increases as expected [21]. However, with the Auston switch ON, the field amplitude increases considerably as the THz probe pulses undergo more passes through the QCL. This indicates clearly that the gain of the cavity is greater than the total losses, and no longer clamped. In figure $2 \mathrm{~g}$, the pulse power amplification at $2.46 \mathrm{THz}$ is $26 \mathrm{~dB}$ after 5 passes through the QCL compared to the value with the Auston switch OFF. Figure 2d (red curve) shows the field of a probe pulse after 7 passes with the Auston switch OFF, and the current density above threshold $\left(178 \mathrm{~A} / \mathrm{cm}^{2}\right)$. This shows that the large amplification with the Auston switch $\mathrm{ON}$ in figure $2 \mathrm{~d}$ (blue curve) arises from un-clamping the gain by an ultra-fast turn-on of the device, and not merely by increasing the current above threshold.

Figures $2 \mathrm{c}$ and $\mathrm{d}$ show that the amplification of the probe pulses eventually saturates as the number of passes through the QCL continues to increase. This occurs because the electrical pulse produced by the Auston switch (and hence the unclamped gain) has a finite time duration. Pulse amplification ceases once the electrical pulse ends, and the gain eventually returns to its clamped value. The finite duration of the electrical pulse can be inferred from Figure 3, where the single pass gain is plotted as a function of the time delay 
between the THz probe pulse and the femtosecond pulse that activates the Auston switch. The gain increases for 60ps, after which it decreases and the net amplification of the probe pulse eventually declines. Each point in figure 3 is a measure of the instantaneous gain convolved with the 18 ps single pass transit time of the probe pulse. The duration of the excess gain and hence the electrical pulse is on the order of 100ps.

The gain does not switch on instantaneously, and the rise time of the gain is found to be approximately 46ps (between the range 10\%-90\%) from figure $3 \mathrm{a}$. This is in rough agreement with a calculated rise time of $26 \mathrm{ps}$ which is derived from the $\mathrm{RC}$ time constant of the calculated $\mathrm{n}^{++}$resistance $(3.2 \Omega)$ and the QCL's capacitance $(3.7 \mathrm{pF})$. The rise time could be significantly reduced by using a metal-metal waveguide [22] which would replace the resistive $\mathrm{n++}$ layer underneath the QCL active region with a metal layer. From figure 3 it can also be observed that for very long times ( $>140 \mathrm{ps)} \mathrm{the} \mathrm{gain} \mathrm{decreases} \mathrm{below} \mathrm{its} \mathrm{value} \mathrm{at}$ negative times. This is caused by an LC resonance and a corresponding voltage oscillation, where the external circuit recharges the common ground of the Auston switch and QCL. (See the supplementary section for further details.)

The gain as a function of the current density with the Auston switch ON (corresponding to $60 \mathrm{ps}$ in figure 3) and with the Auston switch OFF (corresponding to 140ps) is shown in figure 4a. (The current density in Figure 4 is the 'continuous-wave' current density and excludes the current generated by the Auston switch.) With the Auston switch OFF, the gain clamps as expected after threshold $\left(172 \mathrm{~A} / \mathrm{cm}^{2}\right)$ at a value of $12.5 \mathrm{~cm}^{-1}$. With the Auston switch ON the gain is approximately $10 \mathrm{~cm}^{-1}$ greater than the clamped gain, which gives rise to the large amplification observed in figure 2 for the multiple pass pulses. This shows that the transmitted pulses are amplified at the bare cavity gain (i.e. the gain in the absence of the laser field) with the Auston switch ON. The generated ultrafast electrical pulse essentially increases the voltage and current across the QCL before the laser field can build 
up, increasing the gain without being limited to the clamped value. Indeed, an estimate of the current provided by the Auston switch can be found by comparing the current densities for which the center gain is identical for the Auston Switch ON and OFF $\left(\sim 35 \mathrm{~A} / \mathrm{cm}^{2}\right)$ (see also figure 4b) For current densities above threshold, there is a significant internal laser field inside the laser cavity which saturates the bare cavity gain, and reduces the gain to its clamped value at threshold. Thus the excess gain between the bare cavity and clamped gain cannot be used for pulse amplification. This explains the drop in the gain after threshold with the Auston switch ON. (There could also be a contribution from the electric pulse driving the QCL to current densities beyond the maximum power and towards the shut-off current.) We note that this technique also allows the measurement of the maximum bare cavity gain of THz QCLs $\left(22.4 \mathrm{~cm}^{-1}\right.$ for the current device).

Figure $4 \mathrm{~b}$ shows the increase in the center frequency of the gain as a function of current density. With the Auston switch ON (red curve), the center frequency is shifted to higher frequencies than with the Auston switch is OFF (black curve). This frequency shift is most likely the result of a small stark shift of the upper laser levels caused by the QCL being driven to higher biases by the Auston switch. The shift in frequency can also be observed in figure 3 where, before the Auston switch is switched ON, the gain is highest at $2.40 \mathrm{THz}$, whereas the maximum of the unclamped gain peaks at $2.46 \mathrm{THz}$ (at $60 \mathrm{ps}$ ).

Increases in the value of the amplified fields could be obtained; by using QCLs with a greater bare cavity gain (e.g. longitudinal optical phonon based QCLs); by improving the coupling efficiency of the THz pulse into the QCL (currently only on the order of a few percent), and by increasing the length of the QCL cavity (which would reduce losses from the QCL facets). More importantly, the amplification is only limited by the finite duration of the electrical pulses generated by the Auston switch. By increasing their duration by device engineering (see supplementary section), $\mathrm{THz}$ probe pulses could potentially be amplified 
until their fields approach the internal saturation fields of THz QCLs (up to $\sim 1 \mathrm{kV} / \mathrm{cm}$ ). This would effectively injection lock the QCL to the THz probe pulses, and provide a high field source of $\mathrm{THz}$ radiation. This could be then used to generate high-field narrow-band $\mathrm{THz}$ pulses [23, 24] which would be of considerable interest for studying single transitions, nonlinear phenomena and coherent effects in the THz frequency range [25, 26]. Equally, it could be applied to generate high fields over a broad spectral range, using multiple QCL active regions to give a larger gain width [27].

In conclusion, picosecond gain switching of a THz QCL has been demonstrated using an integrated Auston switch. The ultrafast switching placed the laser in a transient state where the gain was no longer clamped, and so could exceed the total losses of the cavity. Consequently, the QCL bare cavity gain was exploited to produce large amplification of $\mathrm{THz}$ pulses from a TDS system. These results show that gain switched QCL amplifiers of THz pulses could significantly advance the current state of $\mathrm{THz}$ technology and extend the applications of THz TDS and QCLs. 


\section{Experimental Methods}

The $\mathrm{THz}$ probe pulses were generated by illuminating an inter-digitated photoconductive antenna with $360 \mathrm{~mW}$ of average power from a $76 \mathrm{MHz}$ femtosecond source (pulse width $80 \mathrm{fs}$, center wavelength $820 \mathrm{~nm}$ ). The inter-digitated GaAs antenna had a gap spacing of $1.5 \mu \mathrm{m}$ and a bias of $4 \mathrm{~V}$ was applied. The $0.5 \mathrm{~mm}$ thick antenna was bonded to a $1 \mathrm{~mm}$ high- resistivity $\mathrm{Si}$ wafer which extended the time between echoes caused by internal reflections. The free-space $\mathrm{THz}$ radiation was collected and refocused onto the sample (and subsequently the electro-optic crystal for detection) with $\mathrm{f \# 2}$ parabolic mirrors. Measurements were taken at $7 \mathrm{~K}$ in a helium flow cryostat. A small $200 \mu \mathrm{m}$ diameter hole in a metal shim was placed in front of the entrance facet of the QCL. A $500 \mu \mathrm{m}<110>\mathrm{ZnTe}$ crystal was used for electro-optic detection of the transmitted $\mathrm{THz}$ pulses. A $2 \mathrm{~mm}<100>$ ZnTe wafer (which has a null electro-optic effect) was attached to the back side of the $<110>$ $\mathrm{ZnTe}$ crystal to extend the time delay of the echoes.

The antenna was modulated at $50 \mathrm{KHz}$ with a duty cycle of $50 \%$. The QCL was modulated at $25 \mathrm{KHz}$ with a $25 \%$ duty cycle, such that every other QCL pulse was co-incident with an antenna pulse. By locking into the modulation frequency of the QCL the difference between the THz electric field with the QCL on and off is measured (the difference field).

The active region of the QCL consisted of 90 periods of $\mathbf{1 . 8} / 12.4$ / 1.5 / 15.3 / $\mathbf{0 . 6}$ / 10.0 / 0.6 / 13.5 / 4.2 / 11.8 / 3.5 / 11.3 / 2.7 / 11.4 / 2.0 / 12.0 / 2.0 / 118.0 layers of GaAs (Al.15 Ga..85As barriers in bold). The 11.4nm and 12.0nm GaAs layers were nominally doped at $1.6 \times 10^{-16} \mathrm{~cm}^{-3}$. The QCL was grown on an undoped GaAs substrate, and the active region was sandwiched between an $80 \mathrm{~nm}$ top and a $700 \mathrm{~nm}$ bottom $\mathrm{n}^{++}$contact layers. An $300 \mathrm{~nm}$ $\mathrm{Al}_{0.5} \mathrm{Ga}_{0.5} \mathrm{As}$ barrier layer separated the lower $\mathrm{n}$-GaAs layer from the undoped GaAs substrate. Devices were processed into a single plasmon geometry with a ridge width of $250 \mu \mathrm{m}$, and the QCLs were mounted onto a Au-coated copper mount to improve heat dissipation. 
The voltage applied to the Auston switch $(27.5 \mathrm{~V})$ was chosen to maximize the output fields of the $\mathrm{THz}$ probe pulses. The large Auston switch voltage is mostly likely do to the large ratio of the QCL capacitance $(3.7 \mathrm{pF})$ to the Auston switch capacitance $(0.16 \mathrm{pF})$ and the $300 \mathrm{~nm}$ thick $\mathrm{Al}_{.50} \mathrm{Ga}_{.50} \mathrm{As}$ etch stop layer between the bottom $\mathrm{n}^{++}$layer and the substrate.

A D.C. voltage was applied across the Auston switch to reduce electrical noise pick up. The Auston switch was excited with $390 \mathrm{~mW}$ of average power from the femtosecond laser. A $200 \mathrm{~mm}$ achromatic lens focused the beam onto the Auston switch, and a $50 \mathrm{~mm}$ divergent cylindrical lens used to expand the beam along the length of the QCL. The resulting beam profile was roughly matched to the area of the Auston switch. A webcam based imaging system verified the laser spot overlapped with the Auston switch. 


\section{References}

[1] Tonouchi, M. “Cutting edge terahertz technology”, Nature Photonics 1, 97 (2007).

[2] Köhler, R. et al. "Terahertz semiconductor-heterostructure laser” Nature 417, 156 (2002).

[3] Kroll, J. et al. "Phase resolved stimulated emission measurements in a laser" Nature 449, 698 (2007).

[4] Jukam, N. et. al. "Investigation of spectral gain narrowing in quantum cascade lasers using terahertz time domain spectroscopy" Appl. Phys. Lett. 93, 101115 (2008).

[5] Koestor, C.J. and Snitzer, E. “Amplification in a fiber laser”, Appl. Opt. 3, 1182 (1964).

[6] Auston, D.H. "Picosecond optoelectronic switching and gating in silicon" Appl. Phys. Lett. 26, 101 (1975).

[7] Chan, W.L. Deibel, J. and Mittleman, D.M. "Imaging with terahertz radiation", Rep. Prog. Phys. 70, 1325 (2007).

[8] Stoik, C.D. Bohm, M.J. and Blackshire, J.L. "Nondestructive evaluation of aircraft composites using transmissive terahertz time domain spectroscopy", Optics Express 16, 17039 (2008).

[9] Shen, Y.C. et al. "Detection and identification of explosives using terahertz pulse spectroscopic imaging" App. Phys. Lett. 86, 241116 (2005).

[10] Dreyhaupt, A. Winnerl, S. Dekorsy, T. and Helm, M. "High intensity terahertz radiation from a microstructure large-area photoconductor" App. Phys. Lett. 86, 121114 (2005).

[11] T. Loffler, T. Hahn, T. Thomson, M. Jacob, F. and Roskos, H.G. "Large-area electrooptic ZnTe terahertz emitters" Optics Express 13, 5353 (2005).

[12] Williams, B.S. “Terahertz quantum cascade lasers” Nature Photonics 1, 517 (2007).

[13] Jukam, N. et al., 'Gain measurements of quantum cascade lasers using terahertz time domain spectroscopy’ IEEE. J. Sel. Topics in Quantum Electronics 14, 436 (2008).

[14] Xu, J. et al. "Tunable terahertz quantum cascade lasers with an external cavity" Appl. Phys. Lett. 91, 121104 (2007).

[15] Mauro, C. "Amplification of terahertz radiation in quantum cascade structures", J. Appl. Phys. 102, 063101 (2007).

[16] Walpole, J.N. "Semicondcutor amplifiers and lasers with tapered gain regions", Optical and Quantum Electronics 28, 623 (1996).

[17] Duguay, M.A. and Damen, T.C. "Picosecond measurement of spontaneous and stimulated emission from injection lasers.” Appl. Phys. Lett. 40, 667 (1982) 
[18] Göbel, O. et al. "Direct gain modulation of a semiconductor laser by a GaAs picosecond optoelectronic switch", Appl. Phys. Lett. 42, 25 (1983).

[19] Paiella, R. et al. "High-speed operation of gain-switched midinfrared quantum cascade lasers" Appl. Phys. Lett. 75, 2536 (1999).

[20] Barbieri, S. "2.9 THz quantum cascade lasers operating up to $70 \mathrm{~K}$ in continuous wave" Appl. Phys. Lett. 85, 1674 (2004).

[21] Parz, W. "Ultra fast probing of light matter interaction in a midinfrared quantum cascade laser” Appl. Phys. Lett. 93, 091105 (2008).

[22] S. Dhillon, S. et al. "Ultralow threshold current terahertz quantum cascade lasers based on double-metal buried strip waveguides", Appl. Phys. Lett. 87, 071107 (2005).

[23] Lee, Y.S. Meade, T. Perlin, V. Winful, H. and Norris, T.B. "Generation of narrow-band terahertz radiation via optical rectification of femtosecond pulses in periodically poled lithium niobate", Appl. Phys. Lett. 76, 2505 (2000).

[24] Danielson, J.R. et al. "Intense band terahertz generation via type II difference-frequency generation in ZnTe using chirped optical pulses", J. Appl. Phys. 104, 033111 (2008).

[25] Cole, B.E. Williams, J.B. King, B.T. Sherwin, M.S. and Stanley, C.R., "Coherent manipulation of semiconductor quantum bits with terahertz radiation" Nature 410, 60 (2001).

[26] Carter, S.G. "Quantum Coherence in an Optical Modulator”, Science 310, 651 (2005).

[27] Freeman, J.R. Marshall, O.P. Beere, H.E. and Ritchie, D.A. "Electrically switchable emission in terahertz quantum cascade lasers" Optics Express 16, 19830 (2008). 


\section{Acknowledgements}

This work was financially supported by the DGA, CNano, ANR, EPSRC and the EC NOTES programme. The LPA-ENS is a "Unité Mixte de Recherche Associée au CNRS UMR8551 et aux Universités Paris 6 et 7". The device fabrication was performed at the nanocenter CTU-IEF-Minerve, which was partially funded by the "Conseil General de l'Essonne".

Materials and correspondence should be addressed to Nathan Jukam at nathan.jukam@1pa.ens.fr

\section{Author Contributions}

Data were taken by N.J., S.S.D, D.O. and J.M., and analyzed by N.J and S.S.D. The experiment was conceived by N.J. Samples were grown by S.P.K., E.H.L., A.G.D., and processed by C.M. S.B. and C.S. The manuscript was prepared by N.J., S.S.D, and J.T. with contributions from S.B., C.S and E.H.L. J.T. and S.S.D supervised and coordinated all work. 


\section{Figure Captions}

Figure 1/ THz Quantum cascade laser with an integrated Auston switch. a, Schematic of the QCL with an Auston switch integrated into one of the side contacts. b, Electrical diagram of the QCL and Auston switch. The QCL is represented as a capacitor and a resistor in parallel. The Auston switch and QCL share a common ground and can be biased independently. c, Pulsed light-current and voltage-current curves of the $2.4 \mathrm{THz}$ QCL at $7 \mathrm{~K}$. The threshold current density is $172 \mathrm{~A} / \mathrm{cm}^{2}$ and maximum power occurs at $184 \mathrm{~A} / \mathrm{cm}^{2}$. d, Schematic of the experimental apparatus.

Figure 2| Fields and spectra of the THz probe pulses with the Austin Switch ON and OFF. The QCL is biased just below threshold at a current density of $168 \mathrm{~A} / \mathrm{cm}^{2} .27 .5 \mathrm{~V}$ is applied to the Auston switch. The blue (black) curves show the difference electric field and spectra with (without) femtosecond laser illumination on the Auston switch. The red curves in $\mathbf{d}$ and $\mathbf{h}$ show the difference electric field and spectra without femtosecond illumination on the Auston switch for a current above threshold at $178 \mathrm{~A} / \mathrm{cm}^{2}$. a-d, The difference electric field of THz probe pulses transmitted through the QCL for a single pass, 3, 5, and 7 passes respectively: e-h show corresponding spectra of the fields.

Figure 3| Duration of the un-clamped gain created by the Auston switch. Single pass gain at $2.40 \mathrm{THz}$ (red), $2.46 \mathrm{THz}$ (green), and $2.53 \mathrm{THz}$ (blue) plotted as a function of the time delay between the $\mathrm{THz}$ probe pulse and the femtosecond laser pulse which turns on the Auston switch. The QCL is biased at a current density of $168 \mathrm{~A} / \mathrm{cm}^{2}$ and the voltage applied to the Auston switch is $27.5 \mathrm{~V}$. (Positive time delays correspond to the Auston Switch pulse arriving before the $\mathrm{THz}$ probe pulse.) 
Figure 4| Gain with the Auston switch OFF and ON - clamped and unclamped gain a, The single pass gain as a function of current density for two different time delays. The red line shows the gain with the Auston switch ON (corresponding to $60 \mathrm{ps}$ in figure 3). The black line shows the gain with the Auston switch OFF (corresponding to $-140 \mathrm{ps}$ in figure 3). The blue dashed box corresponds to the clamped gain. b, The center frequency of the gain as a function of current density with the Auston switch ON (red) and the Auston switch turned OFF (black). The grey shading indicates current densities below threshold. To increase the frequency resolution the data was zero padded. 